\title{
Matrine Restrains Cell Growth and Metastasis by Up-Regulating LINC00472 in Bladder Carcinoma
}

This article was published in the following Dove Press journal:

Cancer Management and Research

\author{
Linlin $\mathrm{Li}^{1, *}$ \\ Fei $\mathrm{Qi}^{1}{ }^{1} *$ \\ Kaichen Wang $\mathbb{D}^{2}$ \\ 'Department of Operating Room, China- \\ Japan Union Hospital of Jilin University, \\ Changchun 130033, Jilin, People's \\ Republic of China; ${ }^{2}$ Department of \\ Urinary Surgery, China-Japan Union \\ Hospital of Jilin University, Changchun \\ 130033, Jilin, People's Republic of China \\ *These authors contributed equally to \\ this work
}

Correspondence: Kaichen Wang Department of Urinary Surgery, China-Japan Union Hospital of jilin University, No. 126

Xiantai Street, Changchun I30033, jilin,

People's Republic of China

$\mathrm{Tel}+86-43 \mathrm{I}-84995999$

Fax +86-43I-89876666

Email wangkc0119@sina.com
Purpose: Bladder Carcinoma (BC) is a malignant carcinoma with a high incidence in masculinity. We preliminarily researched the efficacy and mechanism of matrine (MAT) in T24 and 5637 cells.

Patients and Methods: CCK- 8 , flow cytometry, migration and invasion means were adopted to detect cell viability, apoptosis, migratory and invasive potentials. Moreover, LINC00472 expression was changed via transfection assays and was tested by RT-qPCR. Western blot was used for investigating the levels of CyclinD1, p53, Bcl-2, Bax, pro-Caspase-3, Cleaved-Caspase-3, $\beta$-actin, programmed cell death protein 4 (PDCD4) and relate-proteins of cell pathways. Tumor volume and weight were tested via animal experiments.

Results: MAT could not affect the growth of SV-HUC-1 cell but MAT promoted tumor cell apoptosis but restrained viability, invasion and migration. Furthermore, LINC00472 was prominently low expressed in BC tissues. MAT positively regulated LINC00472 and transfection with si-00472 could partly reverse the efficacies of MAT. Moreover, MAT enhanced PDCD4 expression by up-regulating LINC00472. Besides, we discovered MAT elevated PTEN but restrained PI3K/AKT proteins. Finally, tumor volume and weight were declined by MAT in vivo via up-regulating LINC00472.

Conclusion: MAT restrained cell growth and metastasis but promoted PDCD4 expression by up-regulating LINC00472 via restraining PTEN/PI3K/AKT pathway in BC.

Keywords: bladder carcinoma, matrine, LINC00472, PDCD4

\section{Introduction}

Bladder carcinoma (BC) is a familiar urinary system carcinoma. ${ }^{1}$ Its incidence is higher in masculinity than in femininity. ${ }^{2}$ In recent days, the incidence of BC in China has enhanced year by year. ${ }^{3} \mathrm{BC}$ is an illness that has no distinct symptoms and it is prone to recurrence. ${ }^{4}$ At present, the check means for BC are imaging and urine tests and treatment means are still surgery, radiotherapy and drugs. ${ }^{5}$ In addition, most of the drugs for treating BC lacking specificity and radiotherapy have strong side effects. ${ }^{6}$ Therefore, it is vital to screen safer natural drugs.

Natural products have been recognized as critical latent drugs used for a series of deadly diseases encompassing cancers for a long time. For example, previous studies have pointed out that bitter melon extract (BME) played as a tumor suppressor in breast cancer and neck squamous cell carcinoma (HNSCC), where it inhibited tumor growth by modulation of proliferation, autophagy and immunity. ${ }^{7,8}$ What is more, manifold natural products isolated from Chinese herbal medicine also have been widely utilized for cancer treatment due to their excellent activities, such as anti-proliferation, proapoptosis and anti-metastasis, as well as regulate autophagy and immunity. ${ }^{9}$ Matrine 
(MAT) is a tetracyclic quinolizidine compound extracted from sophora flavescens. ${ }^{10}$ Its molecular formula is $\mathrm{C}_{12} \mathrm{H}_{24}$ $\mathrm{N}_{2} \mathrm{O}$ and its molecular weight is $248.37 \mathrm{~g} / \mathrm{M}^{11}$ It has been put forward that MAT has anti-carcinoma and antiinflammatory efficacies in many diseases. ${ }^{12}$ With the deepening of research, it has been put forward that MAT can conspicuously restrain the activities of carcinoma cells. Researches have pointed out MAT can conspicuously restrain prostate carcinoma. ${ }^{13}$ In addition, a research has pointed out that MAT restrains the activity of BC cells. ${ }^{14}$

Many types of researches have pointed out that the development of malignant carcinomas usually results from abnormal gene expression. ${ }^{15}$ It is widely known that aberrant noncoding RNAs compassing circular (circ) RNAs, long noncoding RNAs (lncRNAs) as well as microRNAs (miRNAs) may have critical latent functions in regulation of gene expression as well as development of cancers. ${ }^{16,17}$ Here, we are interested in the regulation of lncRNAs in BC progress. LncRNAs are a group of RNA more than $200 \mathrm{nt}$ in length. ${ }^{18}$ More and more scientific evidence has proven that IncRNAs are maladjusted in $\mathrm{BC}$, gastric carcinoma and so on, ${ }^{19}$ and it is involved in the occurrence and progress of carcinomas by regulating cell growth and metastasis. ${ }^{20}$ In addition, lncRNAs can regulate several signaling pathways and play a promoting or restraining efficacy in carcinomas. $^{21}$ LINC00472, as a IncRNA, had been put forward to have low expression in colorectal carcinomas ${ }^{22}$ and played a restraining efficacy in breast carcinomas. ${ }^{23}$ However, there was no research about LINC00472 in BC until now.

Currently, we first studied the expression of LINC00472 in BC tissues. T24 and 5637 cells were utilized for the experimental investigation of the efficacy of MAT in vitro. We investigated the efficacies of MAT and LINC00472 on the viability, apoptosis, migration and invasion and the relationship between MAT and programmed cell death protein 4 (PDCD4) was explored. Moreover, we explored the possible mechanism of MAT in BC cells.

\section{Materials and Methods}

\section{Clinical Specimens}

Selected BC tissues and non-tumor tissues $(\mathrm{n}=20)$ were acquired from the patients who had not been treated by any therapies before the cut-off surgery from China-Japan Union Hospital of Jilin University (Changchun, China). Every patient agreed with the performance of this current research and writing an informed consent. This survey was supported by the Medical Ethics Committee of this Hospital.

\section{Cell Culture and Treatment}

BC cells-T24/5637 and the normal bladder cell-SV-HUC-1 provided by American Type Culture Collection (Manassas, VA, USA) were separately hatched at Dulbecco's modified eagle medium (DMEM, Gibco, Grand Island, NY, US) or Ham's F- 12 medium at $37^{\circ} \mathrm{C}$ with $5 \% \mathrm{CO}_{2}$. The streptomycin/penicillin of 100 units $/ \mathrm{mL}$ (Invitrogen, Carlsbad, CA, USA) and 10\% fetal bovine serum (FBS, Gibco) were also added in. MAT (Figure 1, Sigma, St. Louis, MO, US) was diluted with $\mathrm{ddH}_{2} \mathrm{O}$ to IC50 ${ }^{24}$ and was utilized for cells for $24 \mathrm{~h}$ through a filter.

\section{CCK-8}

After dosing dispose, the CCK-8 solution (Sigma) was appended to cells in line with the instructions. Cells were hatched in dark $1 \mathrm{~h}$, the optical density was quantitated at 450 $\mathrm{nm}$ by a microplate reader (Bio-Rad, Sunnyvale, CA, US).

\section{Flow Cytometry}

Transfected cells were harvested and washed with cold $1 \times$ phosphate buffered saline (PBS) after centrifugation. Next, those cells were suspended, followed by $5 \mu \mathrm{L}$ PI and $5 \mu \mathrm{L}$ AnnexinV-FITC staining solution (Bioswamp). After that, the resulting cultures were held at $25^{\circ} \mathrm{C}$ for $10 \mathrm{~min}$ in the dark. Results were measured via flow cytometry via FACS can (Beckman Coulter, Fullerton, CA, USA).

\section{Migration and Invasion Assay}

A 24-well transwell membrane ( $8 \mu \mathrm{m})$-contained chamber (Millipore, Bedford, Massachusetts, USA) was utilized in the migration and invasion experiments. The difference between migration and invasion experiments was the membrane coated with Matrigel (Franklin Lakes, NJ, USA). The transfected BC cells were seeded in the top chamber filling in $200 \mu \mathrm{L}$ serum-free medium, while the lower chamber was filled with complete medium. The intruding chamber was treated for $48 \mathrm{~h}$ to specification. Non-invasive cells were scrubbed. Invading cells were fixed by Methanol, stained by crystal violet and counted by microscope (Olympus, Japan). Randomly selected 5 groups of cells were used for counting.

\section{Transfection}

siRNA targeting LINC00472 (si-00472) and si-negative controls (NC) synthesized by GenePharma Co. (Shanghai, China) were transfected into cells using Lipofectamine 2000 

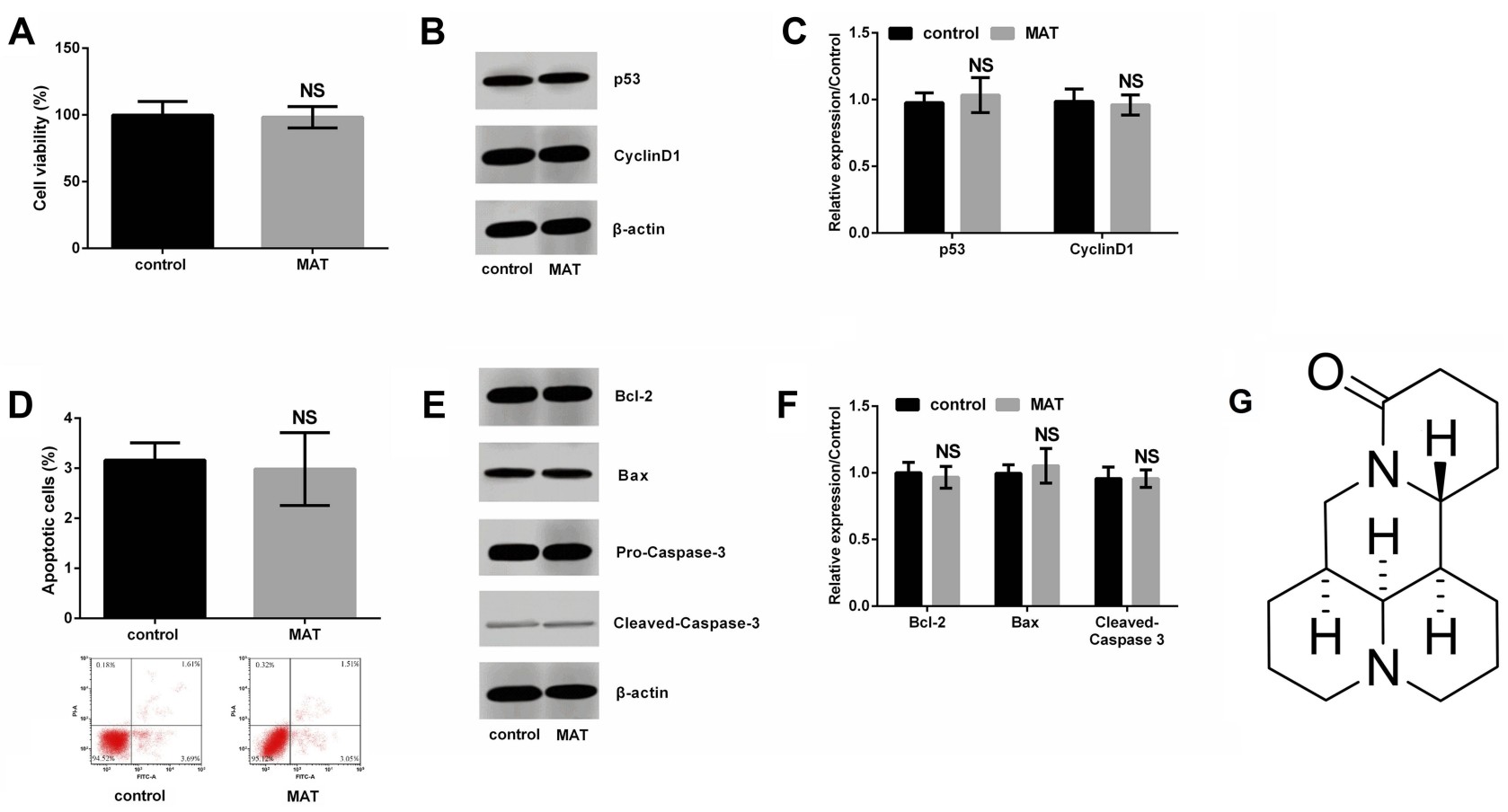

Figure I Matrine (MAT) had no effects on normal SV-HUC-I cell growth. (A) MAT had no effects on viability, and (B, C) generation of p53 and CyclinDI, also (D) apoptotic cells as well as (E, F) expressions of $\mathrm{Bcl}-2$, Bax and Cleaved-Caspase-3; (G) MAT is a tetracyclic quinolizinidine compound. It molecular formula is $\mathrm{C}_{12} \mathrm{H}_{24} \mathrm{~N}_{2} \mathrm{O}$ and molecular weight is $248.37 \mathrm{~g} / \mathrm{mol}$. Pure MAT is white powder, low content is brown and yellow liquor.

Abbreviation: Ns, no significance.

(Invitrogen). Stably transfected cells were selected by G418 medium (Sigma).

\section{RT-qPCR}

Trizol (Invitrogen) was used to extract RNA from BC tissues, non-tumor tissues, or cells. LINC00472 expression was detected by One-Step SYBR ${ }^{\circledR}$ PrimeScript ${ }^{\circledR}$ PLUS RT-RNA PCR Kit (TaKaRa Biotechnology, Dalian, China). $\beta$-Actin was as an internal parameter. The data were calculated as log $10\left(2^{-\Delta \Delta C t}\right)$. Primer sequences used in our experiments were displayed as follows:

LINC00472: Forward: 5'- CCCAGAGACAAGAGGA GCAA-3', Reverse: 5'- AGCGTCAAGAGTGGAGGT TT3'; $\beta$-actin: Forward: 5'-AGCGAGCATCCCCCAAAGT-3'; Reverse: 5'-GGGCACGA AGGCTCATCATT -3'.

\section{Western Blot}

By homogenizing the cells in RIPA buffer (Solarbio), proteins were isolated (Solarbio). Protein assay kit (Bioswamp) was used to quantifying proteins before they were separated by SDS-PAGE and transferred to a PVDF membrane (Millipore). Primary antibodies diluted with 5\% blocking buffer $(1: 1000)$ were cultured the membrane overnight $\left(4^{\circ} \mathrm{C}\right)$. Then, it was washed with PBS and hatched with a secondary antibody goat anti-rabbit IgG (ab6721, Abcam, 1:5000) for $1 \mathrm{~h}$, $25^{\circ} \mathrm{C}$. Signals were developed and examined by the Image $\mathrm{Lab}^{\mathrm{TM}}$ (Bio-Rad). The $\beta$-actin was as internal parameters. Primary antibodies (Abcam) contained antibody CyclinD1 (ab40754), p53 (ab131442), Bcl-2 (ab32124), Bax (ab137321), pro-Caspase-3 (ab32150), Cleaved-Caspase-3 (ab49822), $\beta$-actin (ab8227), PDCD4 (ab51495), phosphate and tension homology deleted on chromosome ten (PTEN) (ab31392), phosphatidylinositide 3-kinases (PI3K)(ab86714), phosphorylated (p)-PI3K (ab182651), protein kinase B (AKT) (ab64148), p-AKT (ab8933).

\section{Animal Experiment}

We performed the in vivo assay by utilizing male athymic BALB/c nude mice (4-6 weeks of age). In detail, mice were divided into 4 groups: control, MAT, MAT + si-NC and MAT + si-00472, of which 8 were in each group. In the control group, the animals were injected intraperitoneally with 100 $\mu \mathrm{L}$ of normal saline the next day after $2 \times 10^{6} \mathrm{~T} 24$ cells were subcutaneously injected into the flank of nude mice. In the MAT group, $50 \mathrm{mg} / \mathrm{kg} /$ day MAT (dissolved in physiological saline) instead of the saline for injection. For MAT + si-NC group: the stable si-NC-transfected T24 cells $\left(2 \times 10^{6}\right)$ were injected into mice, and the next day after inoculation, the animals started an intraperitoneal injection of $50 \mathrm{mg} / \mathrm{kg} /$ day MAT (soluble in physiological saline). For MAT + si-00472 
group: the stably si-00472-transfected T24 cells $\left(2 \times 10^{6}\right)$ were injected before starting the intraperitoneal injection of $50 \mathrm{mg} / \mathrm{kg}$ /day MAT (dissolved in physiological saline). The tumor volume and weight were measured once a week, continuously for 4 weeks.

\section{Statistical Analysis}

Data were shown as mean \pm Standard Deviation (SD). Data analysis was quantified via SPSS 18.0. The $P$-values were calculated by Student's $t$-test or one-way analysis of variance (ANOVA). $P<0.05$ was conspicuously significant.

\section{Results}

\section{MAT Had No Effects on Growth of Normal SV-HUC-I Cells}

We first detected the roles of MAT in normal human uroepithelial cell line-SV-HUC-1. Outcomes were shown in Figure 1, indicating that there were no significant differences in cell viability (Figure 1A) and generation of p53 and CyclinD1 (Figure 1B and C). Also, similar outcomes were observed in apoptotic cells (Figure 1D) as well as expressions of Bcl-2, Bax and Cleaved-caspase-3 (Figure 1E and F) between the control group and MAT treatment group. In addition, the chemical structure of MAT was displayed in Figure 1G. Those data indicated that MAT had no adverse impacts on the normal uroepithelial cell line.

\section{MAT Restrained Cell Growth, Migration and Invasion}

We explored the functions of MAT in T24 and 5637 cells. MAT meaningfully restrained cell viability (Figure 2A, $P<0.01$ or $P<0.001$ ) and promoted apoptosis (Figure 2E, $P<0.001)$. At the molecular level, when MAT was added, Figure 2B-D displayed that the CyclinD1 expression $(P<0.01$ or $P<0.001)$ was conspicuously declined and p53 expression $(P<0.001)$ was conspicuously enhanced.
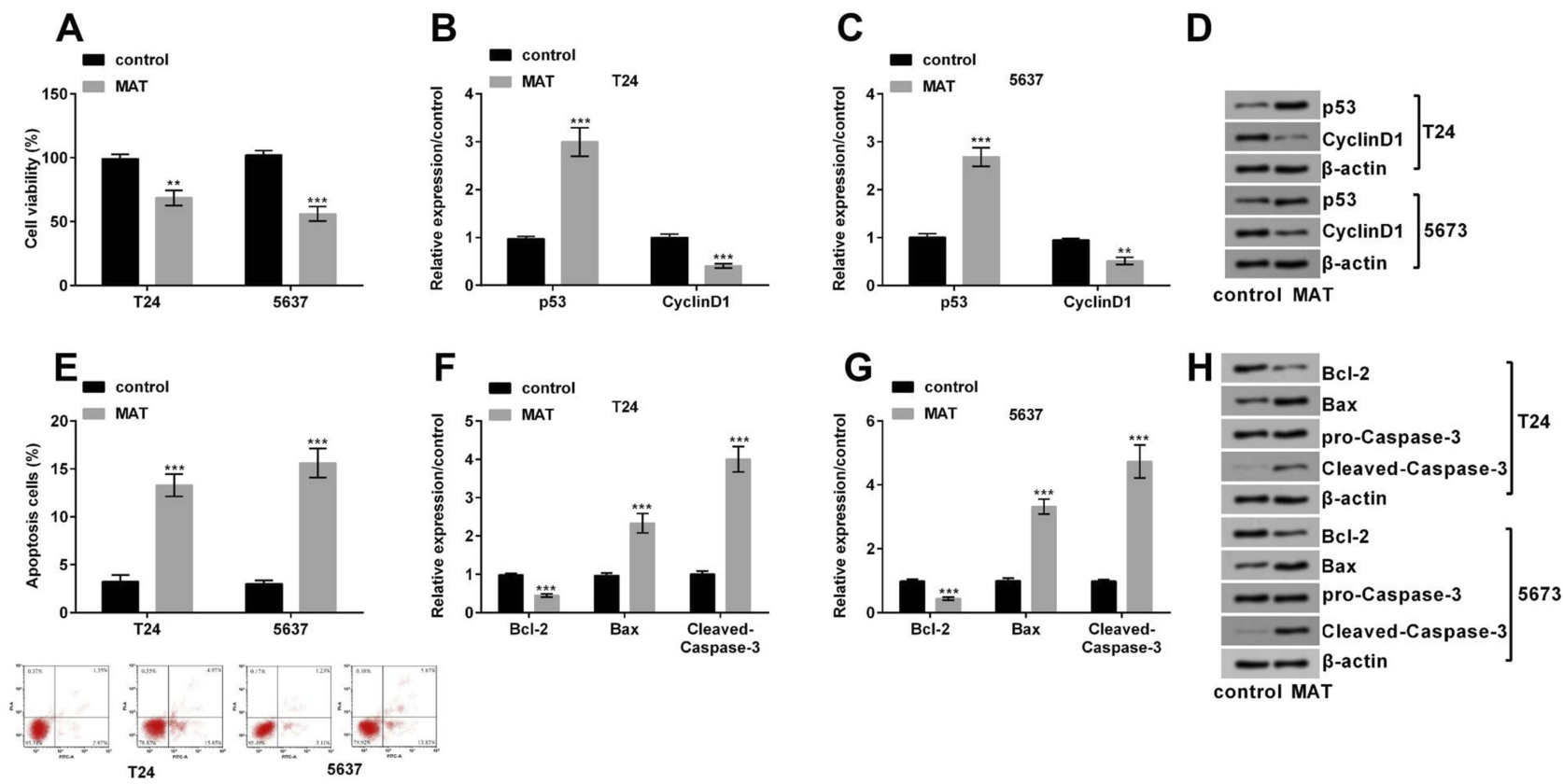

control MAT
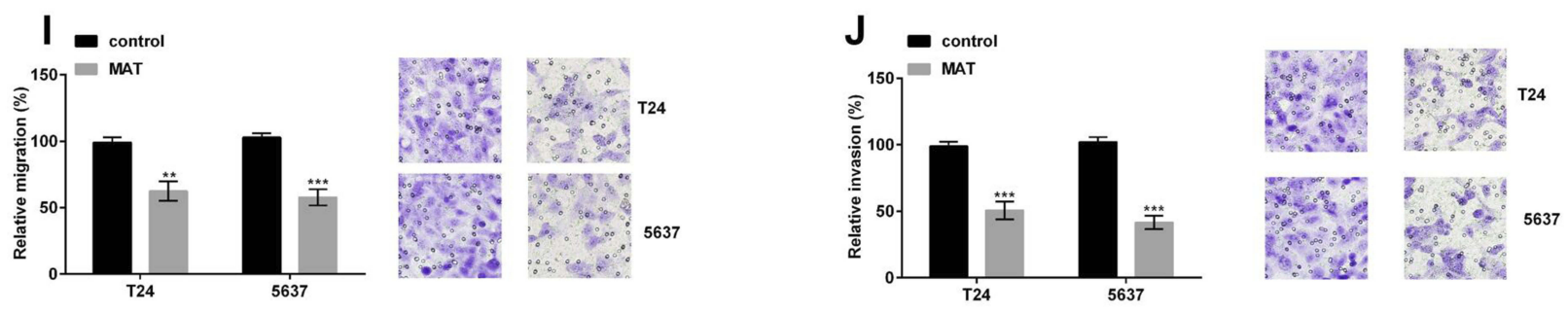

Figure 2 (A) Matrine (MAT) restrained cell growth, invasion and migration of T24 and 5637 cells. MAT restrained cell viability; (B-D) the level of the CyclinDI was conspicuously declined and p53 was conspicuously enhanced when MAT was added; (E) MAT enhanced cell apoptosis; ( $\mathbf{F}-\mathbf{H})$ Bcl-2 expression was conspicuously declined while the levels of Bax and Cleaved-Caspase-3 were conspicuously enhanced when MAT was added; (I) MAT meaningfully restrained cell migration; (J) MAT meaningfully restrained cell invasion. $* * P<0.01 ; * * * P<0.001$. 
Figure $2 \mathrm{~F}-\mathrm{H}$ results pointed out that $\mathrm{Bcl}-2$ expression was conspicuously declined while the levels of Bax and CleavedCaspase-3 were conspicuously enhanced $(P<0.001)$. Furthermore, MAT meaningfully restrained cell migration (Figure 2I, $P<0.01$ or $P<0.001$ ) and invasion (Figure 2J, $P<0.001)$. In conclusion, MAT restrained BC cells' growth, invasion and migration.

\section{MAT Up-Regulated LINC00472}

LINC00472 was detected in BC tissues, corresponding non-tumor tissues, T24 and 5637 cells. The data revealed LINC00472 was prominently low expressed in BC tissues (Figure 3A, $P<0.01$ ). Furthermore, the LINC00472 expression was prominently enhanced when MAT was added (Figure 3B, $P<0.001$ ). This implied LINC00472 was positively regulated by MAT.

\section{MAT Restrained Cell Growth, Migration and Invasion by Up-Regulating LINC00472}

Firstly, we transfected si-NC and si-00472 into T24 and 5637 cells. We put forward si-00472 statistically declined LINC00472 expression (Figure 4A, $P<0.001$ ). Further results revealed that si-00472 disturbed the MAT-induced reduction of viability (Figure 4B, $P<0.05$ ) and improvement of apoptotic cells (Figure $4 \mathrm{C}, P<0.05$ ). At the molecular level, Figure 4D-F displayed that the level of CyclinD1 $(P<$ $0.01)$ was conspicuously enhanced and p53 $(P<0.01)$ was conspicuously declined when transfected si-00472, compared to adding MAT alone. Figure 4G-I pointed out that the levels of Bcl-2 $(P<0.05$ or $P<0.01)$ were conspicuously enhanced while $\operatorname{Bax}(P<0.01)$ and Cleaved-Caspase-3 $(P<0.001)$ were conspicuously declined when transfected si-00472, compared to adding MAT alone. Furthermore, si00472 meaningfully enhanced MAT-induced cell migration
(Figure 4J, $P<0.05$ or $P<0.01$ ) and invasion (Figure $4 \mathrm{~K}$, $P<0.05)$. In short, MAT restrained cell growth, migration and invasion by up-regulating LINC00472 in BC.

\section{MAT Strengthened the Expression of PDCD4 by Up-Regulating LINC00472}

Figure $5 \mathrm{~A}$ and $\mathrm{B}$ revealed that MAT could prominently strengthen the expression of PDCD4 $(P<0.001)$, which was declined by si-00472 $(P<0.01$ or $P<0.001)$. In short, MAT positively regulated PDCD4 expression.

\section{MAT Restrained PTEN/PI3K/AKT Pathways}

In Figure 6A, MAT enhanced PTEN expression and decreased the ratio of $\mathrm{p} / \mathrm{t}-\mathrm{PI} 3 \mathrm{~K} / \mathrm{AKT}(P<0.001)$ while si-00472 declined the MAT-induced rise of ratio of $\mathrm{p} / \mathrm{t}-\mathrm{PI} 3 \mathrm{~K} / \mathrm{AKT}$ in T24 cells $(P<0.05$ or $P<0.01)$. A similar phenomenon appeared in 5637 cells (Figure 6B). In short, MAT might exert its function via restraining PTEN/PI3K/AKT pathways in BC.

\section{MAT Inhibited Tumor Growth in vivo}

In the animal assay, we noticed that compared with the corresponding group, the tumor volume was significantly decreased by MAT treatment separately at $14 \mathrm{~d}(P<0.05), 21 \mathrm{~d}(P<0.01)$ and $28 \mathrm{~d}(P<0.001)$ after injection; however, si-00472 reversed those impacts (Figure 7A). Besides, the tumor weight was declined by MAT treatment $(P<0.01)$, while si-00472 led to an opposite influence $(P<0.05)$ (Figure $7 \mathrm{~B})$. This result indicated that MAT inhibited tumor growth in vivo.

\section{Schematic Diagram of Our Findings}

We designed a schematic diagram for explaining our findings here as shown in Scheme 1. MAT treatment had no effects on SV-HUC-1. But MAT enhanced PDCD4 expression and
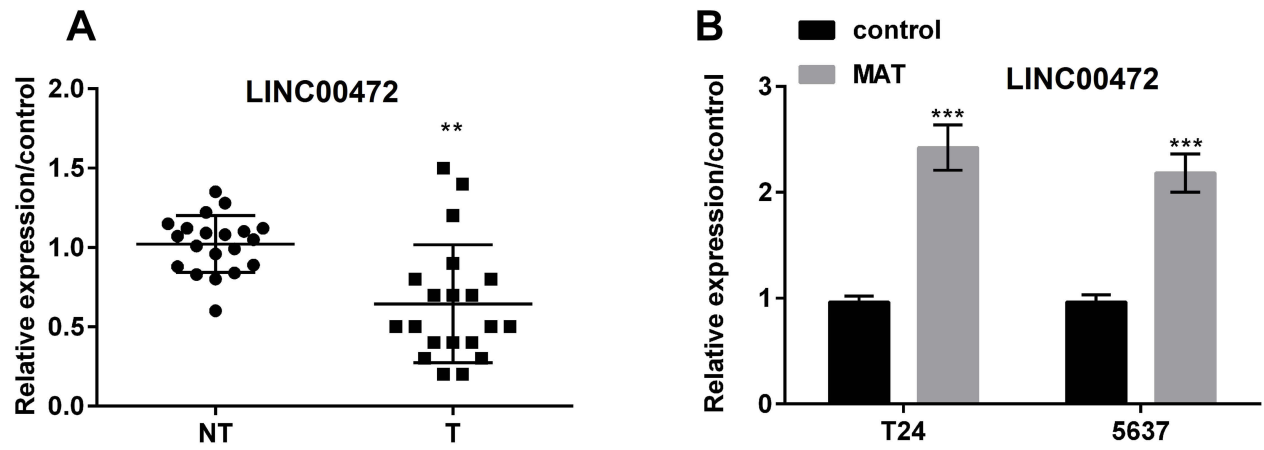

Figure 3 Matrine (MAT) up-regulated long non-coding RNA LINC00472 (LINC00472) in T24 and 5637 cells. (A) LINC00472 expression was prominently lower in BC tissues than in non-tumor tissue; (B) LINC00472 expression was prominently enhanced when MAT was added in T24 and 5637 cells. $* * P<0.0$ I; $* * * P<0.00$ I.

Abbreviations: NT, non-tumor tissue; T, tumor tissue. 

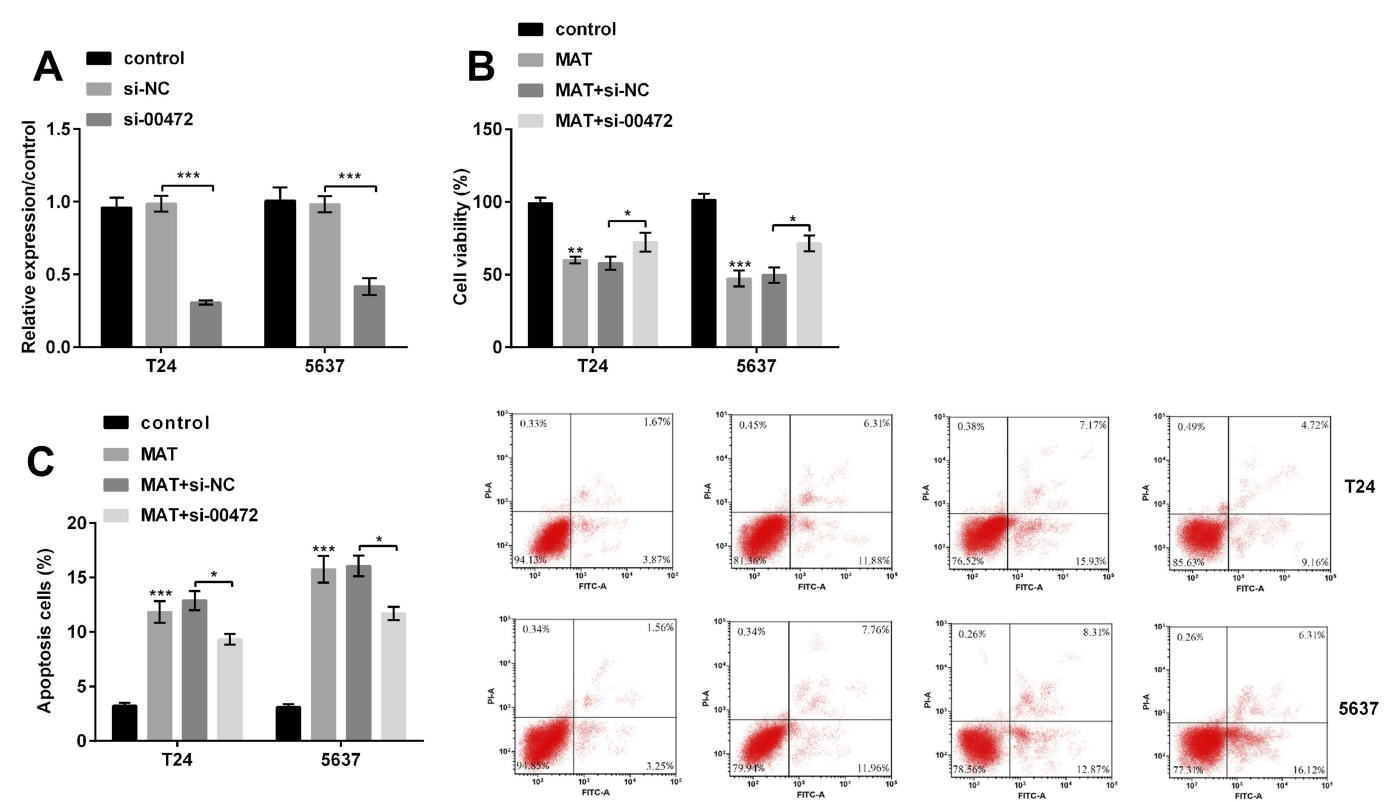
D control
- MAT
- MAT+si-NC

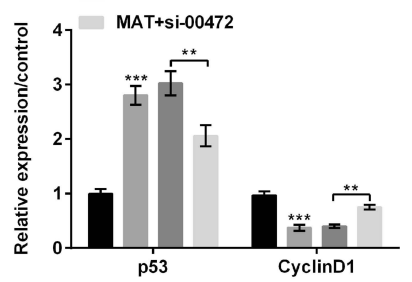

E control

MAT

- MAT+si-NC

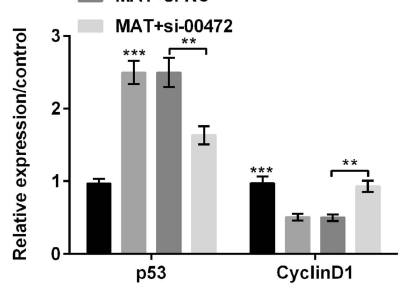

F

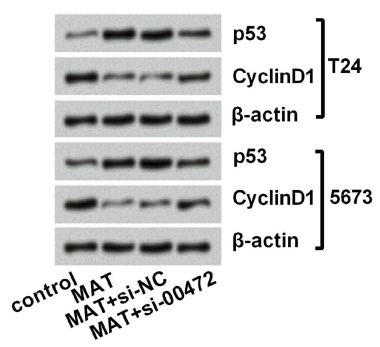
$\mathbf{G}=$ control
- MAT
- MAT+si-NC

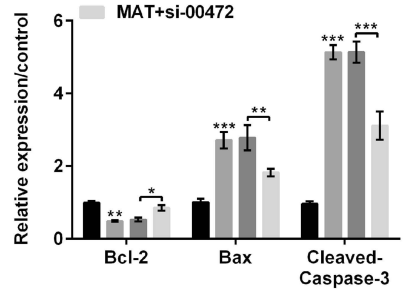

H $=$ control

- MAT

- MAT+si-NC
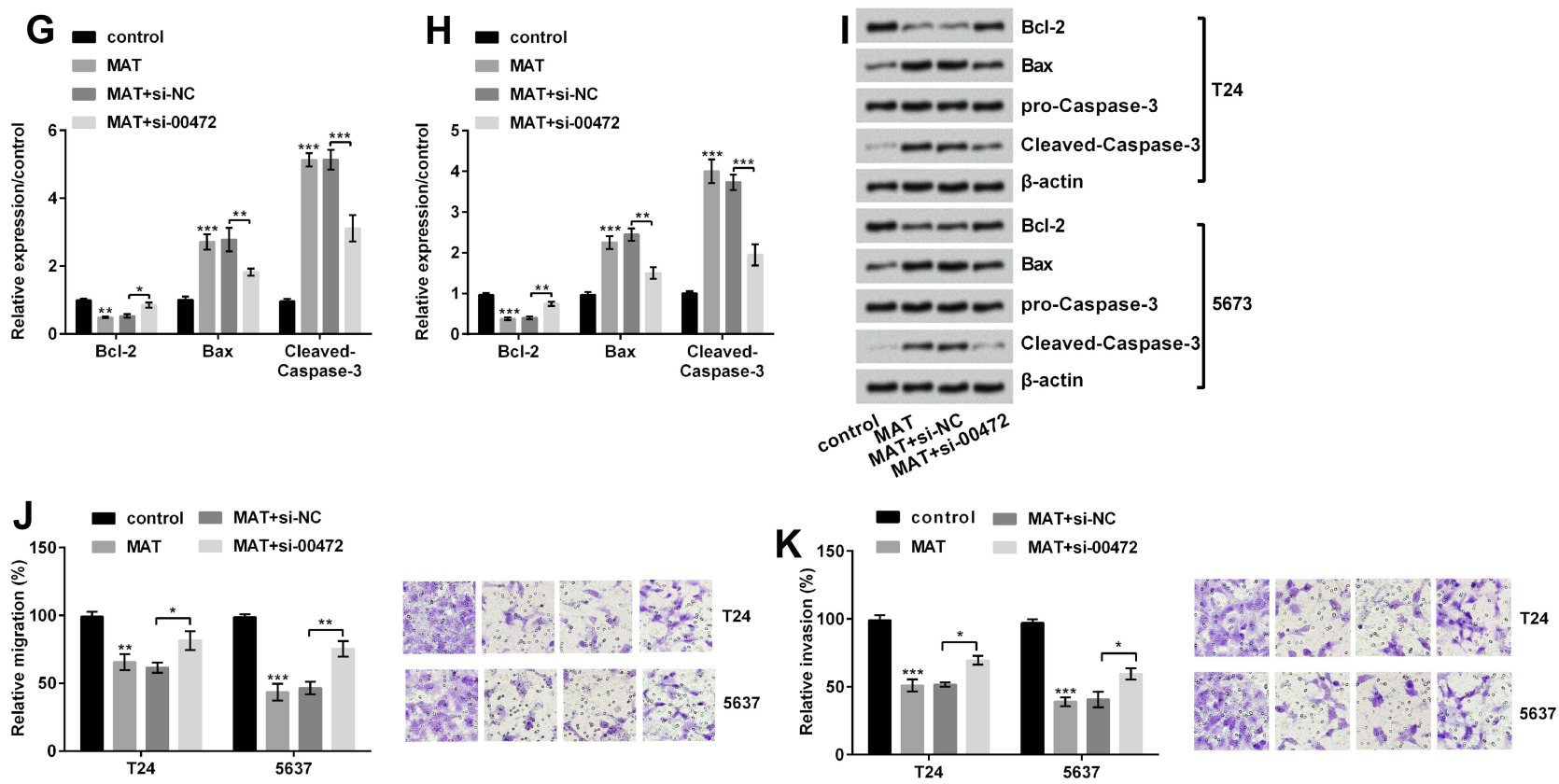

Figure 4 Matrine (MAT) restrained cell growth, migration and invasion but induced apoptosis by up-regulating long non-coding RNA LINC00472 (LINC00472) in T24 and 5637 cells. (A) LINC00472 expression was conspicuously declined by si-00472; (B) si-00472 conspicuously recovered the MAT-inhibited viability; (C) si-00472 meaningfully declined MAT-induced apoptosis; (D-F) the level of CyclinDI was conspicuously enhanced and p53 was conspicuously declined when transfected with si-00472 in cells, compared to adding MAT alone; (G-I) the level of Bcl-2 was conspicuously elevated and Bax and Cleaved-Caspase-3 were conspicuously declined when transfected si-00472, compared to single added MAT alone treatment; (J) si-00472 conspicuously reversed MAT-induced decline in cell migration; (K) si-00472 conspicuously reversed MAT-induced decline in cell invasion. $* P<0.05$; $* * P<0.0$ I; $* * * P<0.00 \mathrm{I}$. Abbreviations: NC, negative control; si-00472, small interference RNA of LINC00472. 


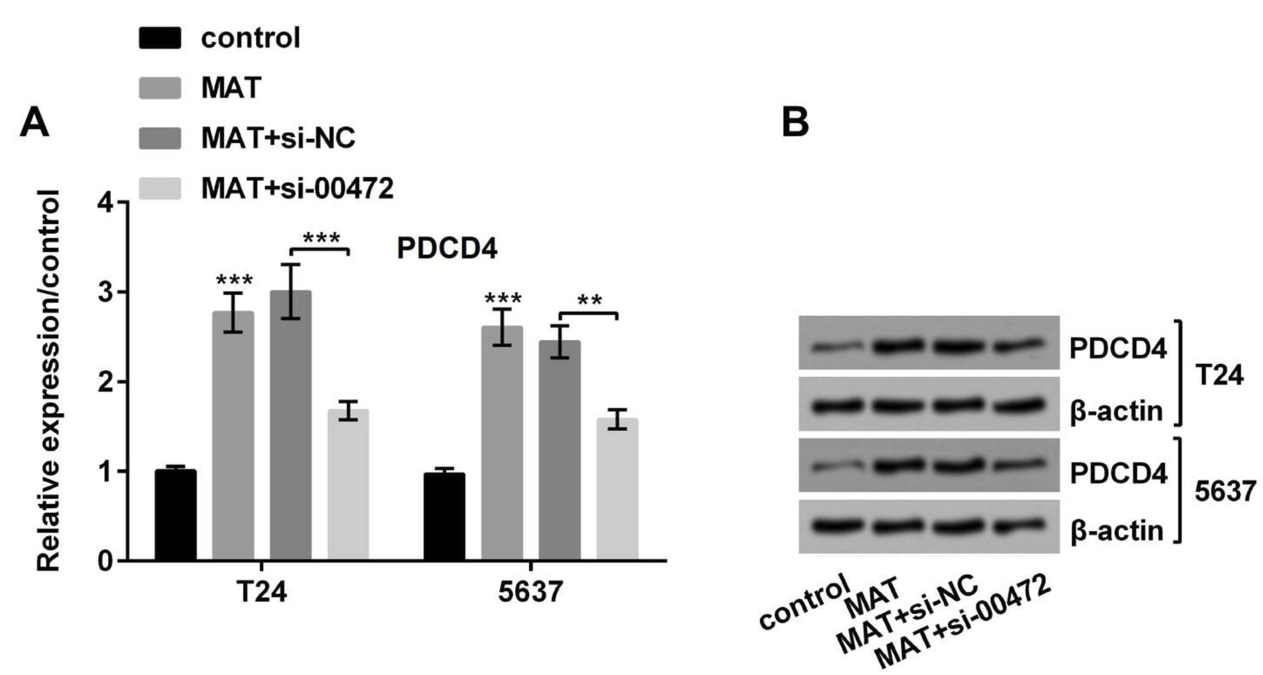

Figure 5 Matrine (MAT) enhanced programmed cell death protein 4 (PDCD4) expressions by up-regulating long non-coding RNA LINC00472 (LINC00472). (A and B) MAT could prominently strengthened the PDCD4, while si-00472 could prominently decline the MAT-induced rise of PDCD4. **P $<0.01 ; * * * P<0.001$. Abbreviations: MAT, matrine; NC, negative control; si-00472, small interference RNA of LINC00472.

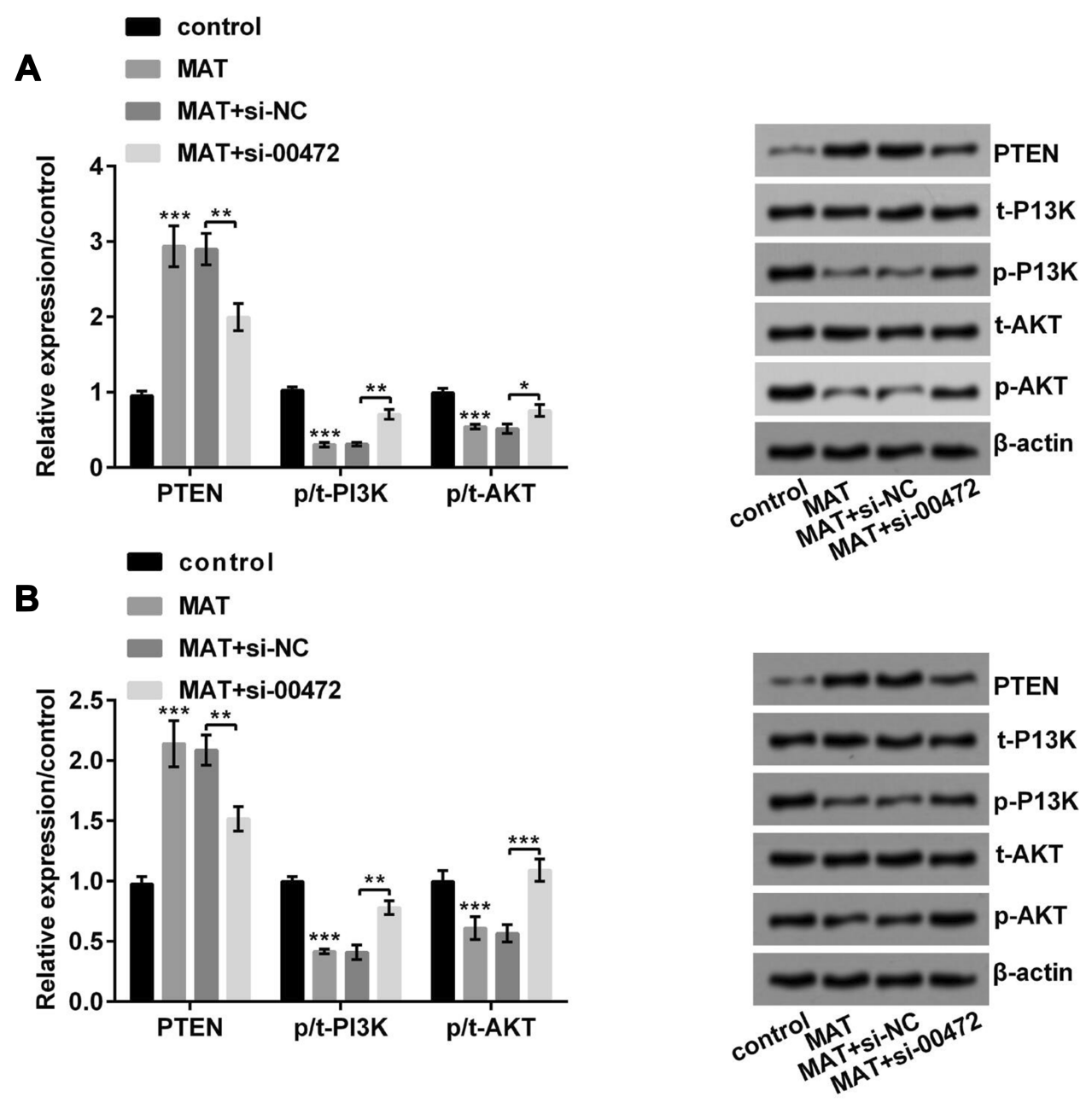

Figure 6 Matrine (MAT) restrained PTEN/PI3K/AKT pathways in T24 and 5637 cells. (A) MAT enhanced PTEN expression and restrained the ratio of p/t-PI3K/AKT in T24 cells; (B) MAT enhanced PTEN expression and restrained the ratio of $p / t-P I 3 K / A K T$ in 5637 cells. $* P<0.05, * * P<0.01$; $* * * P<0.001$.

Abbreviations: PTEN, phosphate and tension homology deleted on chromosome ten; PI3K, phosphatidylinositide 3-kinases; AKT, protein kinase B. 

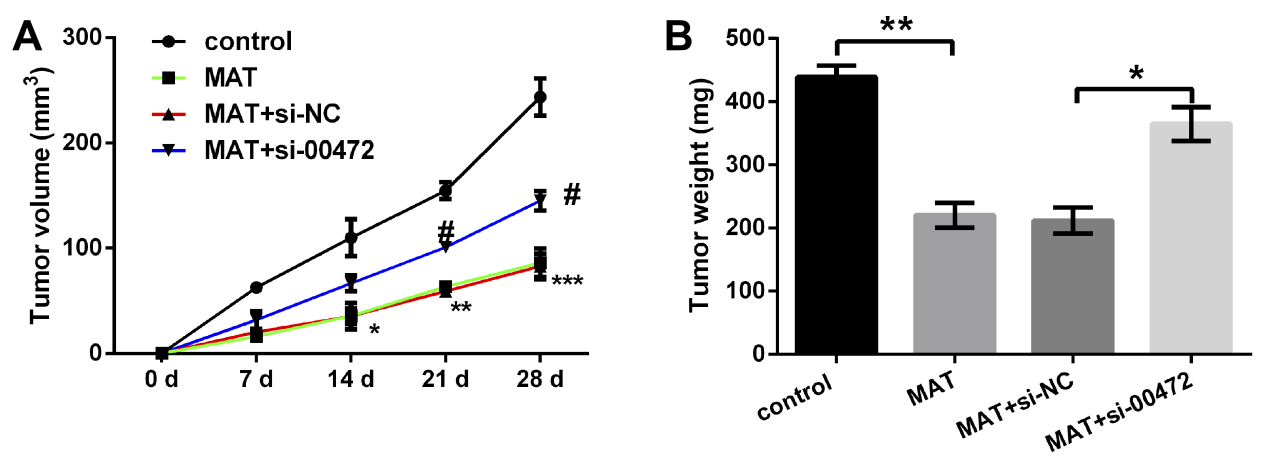

Figure 7 Matrine (MAT) inhibited tumor growth in vivo. (A) The tumor volume was decreased by MAT treatment; (B) tumor weight was reduced by MAT treatment. * $<$ $0.05 ; * *_{p}<0.01 ; * * * p<0.001$, comparing with control; ${ }^{\#} p<0.05$ comparing with si-NC.

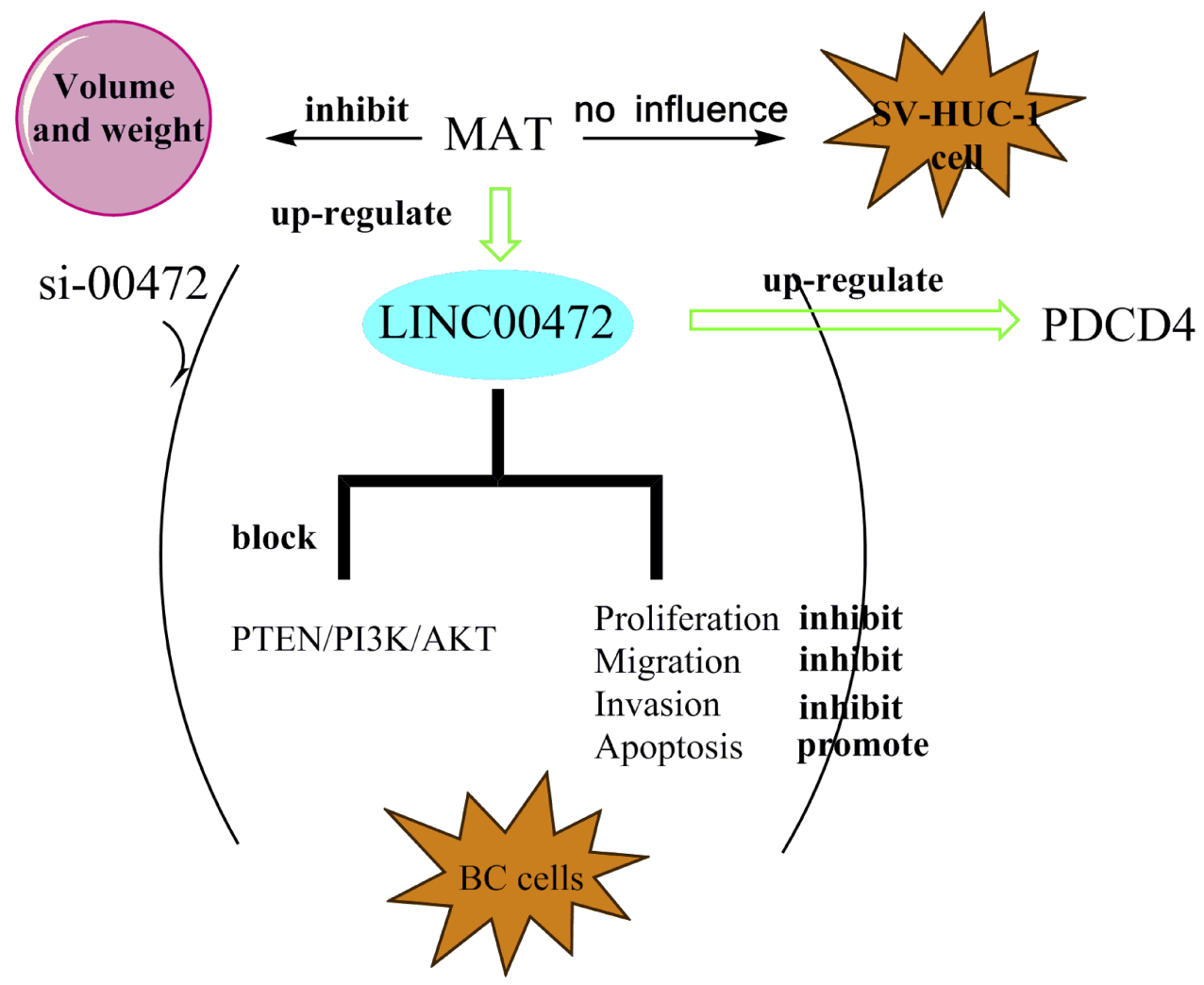

Scheme I Schematic diagram of our findings. A schematic diagram for understanding our findings was made. It showed the anti-tumor roles of MAT in BC. Abbreviations: PTEN, phosphate and tension homology deleted on chromosome ten; PI3K, phosphatidylinositide 3-kinases; AKT, protein kinase B; MAT, matrine; PDCD4, programmed cell death protein 4; BC, bladder Carcinoma.

inhibited $\mathrm{BC}$ cell proliferation, migration, invasion but promoted apoptosis via up-regulating LINC00472 expression by blocking PTEN/PI3K/AKT pathways in vitro. Moreover, MAT treatment restrained tumor growth by reducing tumor weight and volume in vivo.

\section{Discussion}

$\mathrm{BC}$ is a familiar malignant carcinoma in the urinary system. ${ }^{25}$ In this research, we put forward that MAT could restrain the growth, migration and invasion of T24 and 5637 cells, but did not affect normal cell line-SV-HUC-1. LINC00472 was low expressed in bladder carcinoma. In addition, MAT restrained cell growth, migration and invasion by upregulating LINC00472. Further, the research put forward that MAT enhanced PDCD4 expression by up-regulating LINC00472. Finally, we put forward that MAT restrained PTEN/PI3K/AKT pathway and si-00472 reversed MATinduced change in PTEN/PI3K/AKT pathway. Besides, the 
in vivo experiments focused on mice also indicated an antitumor role of MAT. The tumor volume and tumor weight were declined by MAT and were elevated due to LINC00472 knockdown.

As a type of extract of Chinese traditional medicine radix sophorae flavescentis, researches had pointed out that MAT could induce cell apoptosis ${ }^{26}$ and played a protective role in carcinoma. For instance, a research pointed out that MAT restrained cell proliferation in pancreatic carcinoma ${ }^{27}$ while the other research pointed out that MAT restrained cell migration in lung carcinoma. ${ }^{28}$ More interesting, in an animal model of bladder tumor, Gao et al pointed out that MAT could suppress bladder tumor invasion via regulation of the cyclooxygenase-2 (COX-2), and cytosolic recombinant phospholipase A2 (cPLA2). ${ }^{29}$ MAT treatment induced the inhibition of cell proliferation and invasion of bladder cancer, which was mediated by the PI3K/AKT signaling pathway, in vitro. ${ }^{14}$ At the same time, a research displayed that MAT restrained prostate carcinoma, which was another kind of carcinoma of the urinary system. ${ }^{30}$ Unsurprisingly, we also discovered MAT could restrain cell viability, migratory and invasive potentials but promote apoptosis, and played a protective role in $\mathrm{BC}$ cells.

Studies have pointed out that MAT usually plays an anticarcinoma efficacy by regulating RNA. For instance, Wei et al pointed out MAT declined the viability via the miR19b-3p. ${ }^{31} \mathrm{Li}$ et al pointed out MAT exerted anti-carcinoma efficacy in prostate carcinoma via forkhead box O (FOXO). ${ }^{32}$ Furthermore, Liao Li et al pointed out MAT restrained BC cells by up-regulating vascular endothelial growth factor (VEGF) and Wang et al pointed out LINC00472 could be a diagnostic marker in renal tumor. ${ }^{33}$ So we conjectured LINC0047 might be a potential treatment option in BC.

LINC00472 is a newly discovered lncRNA with anticarcinoma effects and usually has lower expression in carcinoma. $^{34}$ For instance, a research had pointed out LINC00472 restrained breast carcinoma cell migration, ${ }^{23}$ and the other research had pointed out LINC00472 enhanced apoptosis in lung carcinoma. ${ }^{35}$ On the other side, a research pointed out that LINC00472 could be as a diagnostic marker in nephropathy. ${ }^{36}$ Unsurprisingly, we put forward MAT positively regulated LINC00472, and si-00472 changed the impacts of MAT on cell viability, apoptosis, migration and invasion. Ulteriorly, we uncovered a research displaying that LINC00472 played a efficacy in carcinoma by promoting PDCD4, ${ }^{37}$ so we further studied the impacts of PDCD4 on $\mathrm{BC}$.
PDCD4 is first discovered in 1994, and it situates in $10 \mathrm{q} 24 .^{38}$ PDCD4 is an anti-carcinoma gene related to cell cycle and apoptosis. ${ }^{39}$ Many researches had pointed out it could be used as a therapeutic target for various carcinomas. ${ }^{40}$ Studies also pointed out that PDCD4 was low expressed in various carcinoma tissues such as gastric carcinoma and so on. ${ }^{41}$ However, the reasons for the decrease of PDCD4 expression levels in carcinomas and their molecular mechanisms were not fully understood. Therefore, we further studied the relationship between MAT, LINC00472 and PDCD4. Gratifyingly, we discovered that PDCD4 was positively regulated by MAT, and MAT enhanced PDCD4 expression by upregulating LINC00472.

The PTEN/PI3K/AKT pathway plays vital efficacies in carcinoma. Previous researches had reported that PTEN/PI3K/AKT pathway participated in the pathogenesis of BC. ${ }^{42}$ In addition, a research had put forward MAT exerted anti-carcinoma roles via PTEN/PI3K/AKT pathway. ${ }^{43}$ Other research pointed out LINC00641 served in $\mathrm{BC}$ via PTEN/PI3K/AKT pathway. ${ }^{44}$ In this paper, the results were completely consistent with those above results. MAT enhanced the expression of PTEN and restrained the ratio of $\mathrm{p} / \mathrm{t}-\mathrm{PI} 3 \mathrm{~K}$ and $\mathrm{p} / \mathrm{t}-\mathrm{AKT}$, while si00472 reversed MAT-induced effects. In short, MAT restrained PTEN/PI3K/AKT signaling pathways by upregulating $\mathrm{LINC00472.}$

\section{Conclusion}

In summary, MAT restrained tumor growth in vivo and abated cell growth, invasion and migration by up-regulating LINC00472/PDCD4 via restraining PTEN/PI3K/AKT pathways in $\mathrm{BC}$ cells. This article might afford a new thought for the clinical treatment of $\mathrm{BC}$.

\section{Ethical Approval}

All procedures performed in studies involving human participants were in accordance with the ethical standards of the institutional committee and with the 1964 Helsinki declaration and its later amendments or comparable ethical standards. The research was ratified by the Medical Ethics Committee of the China-Japan Union Hospital of Jilin University.

\section{Data Sharing Statement}

The datasets used and/or analyzed during the current study are available from the corresponding author on reasonable request.

\section{Acknowledgment}

Linlin Li and Fei Qi are co-first authors for this study. 


\section{Funding}

This research did not receive any specific grant from funding agencies in the public, commercial, or not-forprofit sectors.

\section{Disclosure}

The authors declare that they have no conflicts of interest in this work.

\section{References}

1. Smith AB, Jaeger B, Pinheiro LC, et al. Impact of bladder cancer on health-related quality of life. BJU Int. 2018;121(4):549-557. doi:10.1111/bju.14047

2. Martinez Rodriguez RH, Buisan Rueda O, Ibarz L. Bladder cancer: present and future. Med Clin (Barc). 2017;149(10):449-455. doi:10.1016/j.medcli.2017.06.009

3. He YT, Li DJ, Liang D, et al. [Incidence and mortality of bladder cancer in China, 2014]. Zhonghua Zhong Liu Za Zhi. 2018;40 (9):647-652. doi:10.3760/cma.j.issn.0253-3766.2018.09.002

4. Grayson M. Bladder cancer. Nature. 2017;551(7679):S33. doi:10. 1038/551S33a

5. Bladder cancer: diagnosis and management of bladder cancer: (c) NICE. (2015) Bladder cancer: diagnosis and management of bladder cancer. BJU Int. 2017;120(6):755-765. doi:10.1111/bju.2017.120. issue-6

6. Kaufman DS, Shipley WU, Feldman AS. Bladder cancer. Lancet. 2009;374(9685):239-249. doi:10.1016/S0140-6736(09)60491-8

7. Muhammad N, Steele R, Isbell TS, Philips N, Ray RB. Bitter melon extract inhibits breast cancer growth in preclinical model by inducing autophagic cell death. Oncotarget. 2017;8(39):66226-66236. doi:10.18632/oncotarget.19887

8. Bhattacharya S, Muhammad N, Steele R, Peng G, Ray RB. Immunomodulatory role of bitter melon extract in inhibition of head and neck squamous cell carcinoma growth. Oncotarget. 2016;7(22):33202-33209. doi:10.18632/oncotarget.v7i22

9. Wang S, Wu X, Tan M, et al. Fighting fire with fire: poisonous Chinese herbal medicine for cancer therapy. $J$ Ethnopharmacol. 2012;140(1):33-45. doi:10.1016/j.jep.2011.12.041

10. Michael JP. Indolizidine and quinolizidine alkaloids (July 1999 to June 2000). Nat Prod Rep. 2001;18(5):520-542. doi:10.1039/ b005384h

11. Yong $\mathrm{J}, \mathrm{Wu} \mathrm{X}, \mathrm{Lu} \mathrm{C}$. Anticancer advances of matrine and its derivatives. Curr Pharm Des. 2015;21(25):3673-3680. doi:10.2174/ 1381612821666150122123748

12. Huang J, Xu H. Matrine: bioactivities and structural modifications. Curr Top Med Chem. 2016;16(28):3365-3378. doi:10.2174/ 1568026616666160506131012

13. Huang $\mathrm{H}, \mathrm{Du} T, \mathrm{Xu} \mathrm{G}$, et al. Matrine suppresses invasion of castration-resistant prostate cancer cells by downregulating MMP-2/ 9 via NF-kappaB signaling pathway. Int $J$ Oncol. 2017;50 (2):640-648. doi:10.3892/ijo.2016.3805

14. Yang Y, Guo J-X, Shao Z-Q, Gao J-P. Matrine inhibits bladder cancer cell growth and invasion in vitro through PI3K/AKT signaling pathway: an experimental study. Asian Pac J Trop Med. 2017;10 (5):515-519. doi:10.1016/j.apjtm.2017.05.009

15. Li G-D. Further thoughts on abnormal chromatin configuration and oncogenesis. arXiv Preprint arXiv:1901.04432. 2019. Available from: https://arxiv.xilesou.top/abs/1901.04432.

16. Li Y, Zheng F, Xiao X, et al. CircHIPK3 sponges miR-558 to suppress heparanase expression in bladder cancer cells. EMBO Rep. 2017;18(9):1646-1659. doi:10.15252/embr.201643581
17. Muhammad N, Bhattacharya S, Steele R, Ray RB. Anti-miR-203 suppresses ER-positive breast cancer growth and stemness by targeting SOCS3. Oncotarget. 2016;7(36):58595-58605. doi:10.18632/ oncotarget.v7i36

18. Jarroux J, Morillon A, Pinskaya M. History, discovery, and classification of lncRNAs. Adv Exp Med Biol. 2017;1008:1-46.

19. Peng WX, Koirala P, Mo YY. LncRNA-mediated regulation of cell signaling in cancer. Oncogene. 2017;36(41):5661-5667. doi:10.1038/ onc.2017.184

20. Wang Y, Kong D. Knockdown of IncRNA MEG3 inhibits viability, migration, and invasion and promotes apoptosis by sponging miR-127 in osteosarcoma cell. $J$ Cell Biochem. 2018;119 (1):669-679. doi:10.1002/jcb.26230

21. Hu YC, Wang AM, Lu JK, Cen R, Liu LL. Long noncoding RNA HOXD-AS1 regulates proliferation of cervical cancer cells by activating Ras/ERK signaling pathway. Eur Rev Med Pharmacol Sci. 2017;21(22):5049-5055. doi:10.26355/eurrev_201711_13817

22. Chen L, Zhang W, Li DY, et al. Regulatory network analysis of LINC00472, a long noncoding RNA downregulated by DNA hypermethylation in colorectal cancer. Clin Genet. 2018;93 (6):1189-1198.

23. Liao Q, Shen Y, Katsaros D, et al. Prognostic and predictive values of long non-coding RNA LINC00472 in breast cancer. Clin Genet. 2015;6(11):8579-8592.

24. Liao XZ, Tao LT, Liu JH, et al. Matrine combined with cisplatin synergistically inhibited urothelial bladder cancer cells via down-regulating VEGF/PI3K/Akt signaling pathway. Cancer Cell Int. 2017;17:124. doi:10.1186/s12935-017-0495-6

25. Skeldon SC, Larry Goldenberg S. Bladder cancer: a portal into men's health. Urol Oncol. 2015;33(1):40-44. doi:10.1016/j.urolonc.2013. 12.005

26. Tetik Vardarli A, Duzgun Z, Erdem C, Kaymaz BT, Eroglu Z, Cetintas VB. Matrine induced G0/G1 arrest and apoptosis in human acute T-cell lymphoblastic leukemia (T-ALL) cells. Bosn J Basic Med Sci. 2018;18(2):141-149. doi:10.17305/bjbms.2017.2457

27. Cho YR, Lee JH, Kim JH, et al. Matrine suppresses KRAS-driven pancreatic cancer growth by inhibiting autophagy-mediated energy metabolism. Mol Oncol. 2018;12(7):1203-1215. doi:10.1002/18780261.12324

28. Xie W, Lu J, Lu Q, et al. Matrine inhibits the proliferation and migration of lung cancer cells through regulation of the protein kinase B/glycogen synthase kinase-3beta signaling pathways. Exp Ther Med. 2018;16(2):723-729. doi:10.3892/etm.2018.6266

29. Gao H, Guo Y, Deng N, et al. Suppressive effect of matrine on tumor invasion in N-Butyl-N-(4-Hydroxybutyl)Nitrosamine-induced urinary bladder carcinogenesis. Chemotherapy. 2014;60(2):119-128. doi: $10.1159 / 000371439$

30. Chang J, Hu S, Wang W, et al. Matrine inhibits prostate cancer via activation of the unfolded protein response/endoplasmic reticulum stress signaling and reversal of epithelial to mesenchymal transition. Mol Med Rep. 2018;18(1):945-957. doi:10.3892/ mmr.2018.9061

31. Wei YP, Wang XH, Liu G, et al. Matrine exerts inhibitory effects in melanoma through the regulation of miR-19b-3p/PTEN. Int J Oncol. 2018;53(2):791-800. doi:10.3892/ijo.2018.4414

32. Li Q, Li Q, Huang H, et al. Regulatory effects of antitumor agent matrine on FOXO and PI3K-AKT pathway in castration-resistant prostate cancer cells. Prostate. 2018;61(5):550-558.

33. Wang J, Zhang C, He W, Gou X. Construction and comprehensive analysis of dysregulated long non-coding RNA-associated competing endogenous RNA network in clear cell renal cell carcinoma. 2018.

34. Sui J, Li YH, Zhang YQ, et al. Integrated analysis of long non-coding RNA-associated ceRNA network reveals potential lncRNA biomarkers in human lung adenocarcinoma. Int $J$ Oncol. 2016;49 (5):2023-2036. doi:10.3892/ijo.2016.3716 
35. Su C, Shi K, Cheng X, et al. Long noncoding RNA LINC00472 inhibits proliferation and promotes apoptosis of lung adenocarcinoma cells via regulating miR-24-3p/DEDD. Technol Cancer Res Treat. 2018;17:1533033818790490.

36. Wang YZ, Zhu DY, Xie XM, et al. EA15, MIR22, LINC00472 as diagnostic markers for diabetic kidney disease. J Cell Physiol. 2019;234(6):8797-8803. doi:10.1002/jcp.27539

37. Ye Y, Yang S, Han Y, et al. Linc00472 suppresses proliferation and promotes apoptosis through elevating PDCD4 expression by sponging miR-196a in colorectal cancer. Aging. 2018;10(6):1523-1533. doi:10.18632/aging.v10i6

38. Soejima H, Miyoshi O, Yoshinaga H, Masaki Z. Assignment of the programmed cell death 4 gene (PDCD4) to human chromosome band 10q24 by in situ hybridization. Cytogenet Genome Res. 1999;87(1/ 2):113. doi:10.1159/000015408

39. Matsuhashi S, Manirujjaman M. Control mechanisms of the tumor suppressor PDCD4: expression and functions. Int J Mol Sci. 2019;20 (9):2304.

40. Lankat-Buttgereit B, Goke R. Programmed cell death protein 4 (pdcd4): a novel target for antineoplastic therapy? Biol Cell. 2003;95(8):515-519. doi:10.1016/j.biolcel.2003.09.003
41. Motoyama K, Inoue $\mathrm{H}$, Mimori $\mathrm{K}$, et al. Clinicopathological and prognostic significance of PDCD4 and microRNA-21 in human gastric cancer. Int J Oncol. 2010;36(5):1089-1095. doi:10.3892/ijo_0 0000590

42. Yang X, Cheng Y, Li P, et al. A lentiviral sponge for miRNA-21 diminishes aerobic glycolysis in bladder cancer T24 cells via the PTEN/PI3K/AKT/mTOR axis. Tumour Biol. 2015;36(1):383-391. doi:10.1007/s13277-014-2617-2

43. Wang B, Xu J, Wang H, Chang S, Liu N. Effect and mechanism of sophoridine to suppress hepatocellular carcinoma in vitro and vivo. Biomed Pharmacother. 2017;95:324-330. doi:10.1016/j.biopha.2017. 08.029

44. Li Z, Hong S, Liu Z. LncRNA LINC00641 predicts prognosis and inhibits bladder cancer progression through miR-197-3p/KLF10/ PTEN/PI3K/AKT cascade. Biochem Biophys Res Commun. 2018;503(3):1825-1829. doi:10.1016/j.bbrc.2018.07.120

\section{Publish your work in this journal}

Cancer Management and Research is an international, peer-reviewed open access journal focusing on cancer research and the optimal use of preventative and integrated treatment interventions to achieve improved outcomes, enhanced survival and quality of life for the cancer patient.
The manuscript management system is completely online and includes a very quick and fair peer-review system, which is all easy to use. Visit http://www.dovepress.com/testimonials.php to read real quotes from published authors. 